Special Article

\title{
Common issues in the management of patients in the waiting list and after liver transplantation
}

\author{
Patrizia Burra $^{a, *}$ Luca Saverio Belli $^{\mathrm{b}}$, Stefano Ginanni Corradini ${ }^{\mathrm{c}}$, Riccardo Volpes ${ }^{\mathrm{d}}$, \\ Marco Marzioni ${ }^{\mathrm{e}}$, Edoardo Giannini $^{\mathrm{f}}$, Pierluigi Toniutto ${ }^{\mathrm{g}}$ \\ a Multivisceral Transplant Unit, Department of Surgery, Oncology and Gastroenterology, Padova University Hospital, Italy \\ b Department of Hepatology, Niguarda Hospital, Milan, Italy \\ ' Gastroenterology, La Sapienza University, Rome, Italy \\ ${ }^{\mathrm{d}}$ Hepatology and Gastroenterology Unit, ISMETT-IRCCS, Palermo, Italy \\ e Gastroenterology, University Hospital, Ancona, Italy \\ ${ }^{\mathrm{f}}$ Gastroenterology, University Hospital, Genova, Italy \\ g Internal Medicine, University Hospital, Udine, Italy
}

\section{A R T I C L E I N F O}

\section{Article history:}

Received 28 September 2016

Received in revised form 19 December 2016

Accepted 22 December 2016

Available online 31 December 2016

\section{Keywords:}

Alcoholic liver disease

DAAs

HBV recurrence

Immunosuppression

Liver transplantation

\begin{abstract}
A B S T R A C T
The present document contains the recommendations of an expert panel of transplant hepatologists, appointed by the Italian Association for the Study of the Liver (AISF), on how to manage the most common aspects of liver transplantation: the topics covered include: new treatments for HCV in patients on the waiting list for liver transplantation; antiviral treatments in patients with HCV recurrence after liver transplantation; prophylaxis for HBV recurrence after liver transplantation; indications for liver transplantation in alcoholic liver disease; and Immunosuppressive therapy. The statements on each topic were approved by participants at the AISF Transplant Hepatologist Expert Meeting (organized by the Permanent Committee on Liver Transplantation in Mondello on 4-5 October 2015), and are graded according to the Oxford classification of levels of evidence.
\end{abstract}

(C) 2016 Editrice Gastroenterologica Italiana S.r.l. Published by Elsevier Ltd. All rights reserved.

\section{Introduction}

The present document contains the recommendations of an expert panel of transplant hepatologists, appointed by the Italian Association for the Study of the Liver (AISF), on how to manage the most common aspects of liver transplantation. The method used to develop these recommendations involved the following steps: the 5 members of the AISF Permanent Committee on Liver Transplantation chose the following main topics of interest: 1 . New treatments for HCV in patients on the waiting list for liver transplantation; 2. Antiviral treatments in patients with $\mathrm{HCV}$ recurrence after liver transplantation; 3. Prophylaxis for HBV recurrence after liver transplantation; 4. Indications for liver transplantation in alcoholic liver disease; and 5. Immunosuppressive therapy. For each topic, a working group was selected by the members of the AISF committee that consisted of several experts plus a chairman who was one of the 5 members of the AISF committee. In all, 36 trans-

\footnotetext{
* Corresponding author.

E-mail address: burra@unipd.it (P. Burra).
}

plant hepatologists (experts) were invited to join the groups. They were chosen on the grounds of their competence, role, expertise and publications/research in the field of end-stage liver disease and liver transplantation. The experts involved in the 5 groups are listed in Appendix A. For each group, the chairman identified the relevant questions regarding clinical practice and controversial issues, which were then circulated within each working group to refine the topics and avoid duplications. The working groups independently developed their recommendations, and conducted a systematic literature search and review (using Medline/PubMed) to support all definitions and statements, grading each recommendation according to the Oxford classification of levels of evidence [1]. All working group participants and members of the Permanent Committee on Liver Transplantation took part in the AISF Transplant Hepatologist Expert Meeting organized by the committee in Mondello on 4-5 October 2015. During the first sessions at the meeting, the chairmen of the working groups presented the 5 topics, and the recommendations their group had developed on each question. This was followed by a general debate to refine the recommendations, making any necessary adjustments. At the end of this discussion, each working group met to finalize their position statements in the 
light of the input they had received. At a final general session, the working group chairmen submitted each recommendation to the approval of all 41 participants at the meeting.

\section{Antiviral treatment for HCV-positive patients on the waiting list and after liver transplantation (LT)}

HCV infection is the leading cause of death due to liver disease, and the most common indication for LT in the US and Europe [2,3]. Recurrent HCV infection in the graft was judged to affect all patients who undergo LT and have active HCV replication [4]. HCV-positive recipients experience a more accelerated progression of liver damage and onset of significant liver fibrosis than immune-competent patients [5], and about $30 \%$ of them develop graft cirrhosis within 5 years after LT. Severe HCV recurrence is responsible for the worse outcome in HCV-positive recipients than in patients undergoing LT for other indications [6].

The treatment options for HCV-related liver disease have been evolving rapidly since the approval of the first direct antiviral agents (DAAs), Boceprevir and Telaprevir, in 2011. The new DAAs that became available as of 2014 target different HCV genome regions: NS3, NS5A and NS5B. The NS3 inhibitors already available are Simeprevir and Paritaprevir, while Grazoprevir is expected to become available soon. The NS5A inhibitors currently available are Ledipasvir, Daclatasvir and Ombitasvir, while Elbasvir and Velpatasvir will also be available soon. Two distinct NS5B inhibitors, Sofosbuvir and Dasabuvir, are currently used in combination with NS3 and/or NS5A inhibitors.

The new DAAs are very effective and well tolerated. They have completely changed the picture of anti-HCV therapy. The vast majority of HCV-positive patients with severe liver disease can now be treated successfully either pre- or post-LT. Given their favorable profile, two different approaches can now be pursued:

- treating HCV infection before LT, while the patient is on the waiting list. This policy has some clear advantages in terms of preventing liver graft reinfection, improving liver function (to such an extent that some patients may be delisted), and facilitating post-LT management;

- treating HCV infection after LT, either soon afterwards to take advantage of the removal of the infected native liver and the consequently very low viral burden, or at the time of $\mathrm{HCV}$ recurrence, as done in the past.

\subsection{Question 1}

Which DAA or combination of DAAs can be used in cirrhotic patients listed for LT, and what are the expected sustained virological response (SVR) rates while on the list?

\subsubsection{Statement}

1a. Sofosbuvir, Ledipasvir, and Daclatasvir can be used in patients with cirrhosis with no need for dose adjustments whatever the functional impairment of the liver (1), $\mathbf{A})$.

1b. The 3D combo (Paritaprevir/r, Ombitasvir, Dasabuvir), and 2D combo (Paritaprevir/r, Ombitasvir) should not be used in patients with moderate-to-severe hepatic impairment (Child-Pugh B and C). Simeprevir is not recommended in patients with moderate hepatic impairment (Child-Pugh B), and should be avoided in Child-Pugh C (1b, A). The 3D or 2D combo, and Simeprevir can be safely used in Child-Pugh A (1), A).

1c. Since Sofosbuvir requires dose adjustments in cases of an eGFR $<30 \mathrm{~mL} / \mathrm{min}$ (every other day), and in patients on hemodialysis, it is not yet licensed in Italy for use in these clinical conditions [4]. In cases of severe kidney impairment, treatment with Sofosbu- vir (if licensed) should preferably be administered after LT. The 3D and 2D combo can be used in patients with an eGFR $<30 \mathrm{~mL} / \mathrm{min}$, but only if liver function is preserved (Child-Pugh $A$ ).

1d. Virological response after DAA therapy is very high, in the order of $90 \%$, in patients with compensated cirrhosis (Child-Pugh A) and high, in the order of $80 \%$, in those with decompensated cirrhosis (Child-Pugh B-C). DAA therapy can therefore be considered for patients listed for LT (1), A).

\subsubsection{Comment}

DAAs should be used with caution in LT candidates with severely impaired liver function (Child-Pugh B and C), or severe renal dysfunction (eGFR $<30 \mathrm{~mL} / \mathrm{min}$ ), because both of these conditions affect the metabolism of some DAAs [7]. In Italy, the AIFA (Agenzia Italiana del Farmaco) warns against the use of Sofosbuvir if the eGFR is $<30 \mathrm{~mL} / \mathrm{min}$.

\subsection{Question 2}

What is the impact of DAAs on liver function and inactivation/delisting?

\subsubsection{Statement}

2a. In Child B patients, a significant improvement in Child-Pugh ( $>2$ points) or MELD score ( $>3$ points) can be achieved in $20-40 \%$ of cases. Although this issue is still largely unexplored, preliminary data indicate that delisting is possible in at least $15 \%$ of treated patients [8]. Further studies are also needed to identify possible predictors of delisting, and which patients might benefit most from DAA treatment $(\mathbf{4}, \mathbf{C})$.

2b. A similar significant improvement in Child-Pugh or MELD score can be achieved in Child Pugh C patients with a MELD score $<25$, but this improvement may not suffice to enable their delisting, and it may hamper their access to LT. Further studies are required to ascertain to what extent DAA may be beneficial or detrimental to Child-Pugh $C$ patients from an intention-to-transplant perspective $(4, C)$.

\subsubsection{Comment}

Excellent SVR rates and a very good safety profile can now be achieved with the new DAAs, which have virtually no contraindications, even in decompensated patients on the waiting list. Interestingly, improvements in liver function (albumin and bilirubin levels) and measures of decompensation - including the MELD and Child-Pugh scores - have been reported in patients with advanced liver disease during and shortly after treatment with Sofosbuvir/Ledipasvir [9-11], or Sofosbuvir/Daclatasvir [11-14]. There is still some uncertainty, however, as to whether there is a point of no return, whether this therapy is safe in patients with advanced liver insufficiency, and whether short-term positive effects on hepatic decompensation will translate into long-term clinical benefits [15].

\subsection{Question 3}

What is the impact of pre-LT treatment on post-LT HCV recurrence?

\subsubsection{Statement}

3a. Based on current knowledge, the prevention of post-LT HCV recurrence thanks to pre-LT DAA treatment requires a period of virological suppression of at least 30 days. Patients should therefore be placed "on hold" until this goal has been reached, providing their expected waiting time is long enough (2b, B). 


\subsubsection{Comment}

So far, this issue has only been addressed in one study by Curry et al. [16], who showed that pre-LT DAA therapy can effectively prevent HCV recurrence in 95\% of Child-Pugh A patients, providing a minimum 30-day period of viral suppression before LT is granted. Though nobody yet knows whether the same results can be achieved in patients with decompensated cirrhosis (Child-Pugh B and C), data from the Solar 1 and 2 studies [2,3] are encouraging: in about $80 \%$ of patients with decompensated cirrhosis, 4 weeks of treatment with Sofosbuvir/Ledipasvir and Ribavirin can result in an HCV-RNA below the level of detection.

\subsection{Question 4}

Who should or should not be treated before LT?

\subsubsection{Statement}

4a. Based on current research and practice, pre-LT DAA treatment is not recommended in patients with high MELD scores $(>25)$ because of safety concerns and their rapid access to LT. Post-LT DAA treatment is preferable in these patients $(\mathbf{5}, \mathbf{D})$.

4b. In Child-Pugh B patients, the likelihood of clinical improvement and a regression of the signs of decompensation makes DAA treatment before LT a reasonable option because some of these patients may be delisted. In Child-Pugh $C$ patients with a MELD $<25$, the decision whether to treat should be made with caution to ensure that it will not be detrimental to the patient $(\mathbf{4}, \mathrm{D})$.

4c. Among patients listed with hepatocellular carcinoma (HCC), pre-LT treatment should be restricted to those expected to have to wait $>3$ months for LT $(\mathbf{5}, \mathbf{D})$.

\subsubsection{Comment}

The following factors should be considered when deciding whether to treat patients before or after LT:

- the urgency of the case;

- the chances of delisting if the primary indication for LT is decompensated cirrhosis without HCC;

- cost-effectiveness considerations.

Being aware of these factors helps futile DAA treatments to be avoided.

Finally, recent conflicting evidence of a higher risk of HCC recurrence when DAA are given to patients previously treated for HCC needs to be explored in appropriately designed studies $[17,18]$.

\subsection{Question 5}

Is bridging therapy a valid option?

\subsubsection{Statement}

5a. Bridging therapy cannot be recommended on a routine basis because of a lack of data on the issue. It might be considered in the event of an unexpected, rapid deterioration in liver function due to incidental events while on DAA therapy, particularly in patients who are still viremic. Any decision to continue DAA treatment across transplant should take into account early liver graft function, postoperative renal function, and drug-to-drug interactions (5, D).

\subsubsection{Comment}

Very limited data are available on DAA absorption early after the surgical procedure, when liver function may be suboptimal due to ischemic reperfusion injury or an unexpectedly poor graft quality [19]. Another reason for caution concerns the potential interactions between the many drugs needed in the early post-operative period, and because of the risk of acute renal failure.

\section{Antiviral treatment in patients with $\mathrm{HCV}$ recurrence after liver transplantation}

Data emerging from registration trials and clinical practice clearly demonstrate that antiviral strategies involving new DAAs have achieved a SVR in more than $95 \%$ of LT recipients with recurrent HCV infection. This means we are now in a "new DAA era" that will substantially change our approach to the burden of HCV in the post-LT setting. The issues that remain to be clarified concern drugto-drug interactions between DAAs and immunosuppressants, and the safety profile of DAAs in patients with advanced liver disease and/or renal insufficiency. These aspects are of paramount importance in HCV-positive LT recipients because most of them develop renal impairments post-LT due to calcineurin inhibitor toxicity.

\subsection{Question 6}

What are the main determinants for deciding when to start antiviral therapy for HCV recurrence?

\subsubsection{Statement}

6a. All patients should be treated as early as possible after LT. Patients who experience recurrent HCV infection with fibrosing cholestatic hepatitis should take higher priority.

Antiviral treatment should be started taking the following issues into account:

- clinical conditions should be stable and patients should be out of the intensive care unit;

- serum immunosuppressant levels should be stable;

- renal function must allow for the use of DAAs $(5, \mathbf{D})$.

6b. In patients who develop decompensated graft cirrhosis due to $\mathrm{HCV}$ recurrence, the first option to consider is retransplantation. Antiviral therapy should only be offered as a first option to patients with contraindications for retransplantation whose advanced liver disease represents their main risk of death $(\mathbf{5}, \mathbf{D})$.

\subsubsection{Comment}

Before the advent of DAAs, the recommended time to start antiviral treatment for recurrent HCV infection depended on a patient's persistently elevated alanine aminotransferase levels, and on histologically-confirmed significant graft fibrosis, after rejection, biliary obstruction, and vascular damage had been ruled out. Interferon-based antiviral therapy was associated with high rates of side effects that required dose reductions or premature interruptions of the treatment in the majority of patients, as well as carrying a risk of graft rejection. Early severe HCV recurrences, w fibrosing cholestatic hepatitis, were associated with higher mortality rates and a less effective antiviral treatment. Nowadays, with the new DAAs, these conditions can be treated with good outcomes, as Forns et al. demonstrated [20]: given Sofosbuvir-based antiviral therapy, their patients with early HCV recurrence were more likely to achieve a SVR12 (73\%) than patients with established cirrhosis (43\%); and a larger proportion of the former patients showed clinical improvements in ascites and hepatic encephalopathy than among the patients with decompensated cirrhosis (69\% vs. $45 \%$, respectively). These results suggest that early DAA treatment for patients with recurrent HCV infection after LT may offer an advantage over waiting until patients develop more advanced fibrosis. Secondly, treating HCV infection during the first weeks after LT (i.e. within 30 days) could help to prevent HCV extrahepatic dissemination. It is well known that HCV infection is associated 
with injury to organs other than the liver, leading to the onset of HCV-related extra-hepatic manifestations believed to contribute to higher morbidity and mortality rates [21]. In this setting, early $\mathrm{HCV}$ eradication may protect against the clinical consequences of extra-hepatic manifestations such as cryoglobulinemic vasculitis, glomerulonephritis and polyneuropathy, as well as lymphoma and diabetes. On the other hand, deferring antiviral treatment for HCV recurrence could favor the onset of irreversible organ injury [22]. Bearing in mind that a SVR12 is still unsatisfactory in DAA-treated patients with decompensated liver cirrhosis due to $\mathrm{HCV}$ recurrence, the retransplantation option should be considered first $[9,11]$.

\subsection{Question 7}

Should per protocol liver biopsies be maintained in the surveillance of liver fibrosis progression?

\subsubsection{Statement}

7a. Other than for scientific research purposes, no per protocol liver biopsies are indicated in patients who achieve a SVR and have no signs of liver injury $(\mathbf{3 b}, \mathbf{C})$.

7b. Liver biopsy remains an important tool for the histological assessment of abnormal liver function (1c, $\mathbf{A})$.

\subsubsection{Comment}

Per protocol liver biopsy has traditionally been considered the reference method for assessing tissue damage and the progression of hepatic fibrosis in patients with recurrent HCV infection [23]. Pathologists have developed robust scoring systems for staging liver fibrosis, such as the semi-quantitative METAVIR score [24], but several non-invasive tests for assessing the severity of graft fibrosis have been introduced in clinical practice in recent years. Gambato et al. [25] showed that measuring liver stiffness is useful both for stratifying patients by their risk of progression and for predicting HCV-related outcomes in recurrent hepatitis C. Bignulin et al. likewise demonstrated that measuring the acoustic radiation force impulse is highly accurate in ruling out significant graft fibrosis due to HCV recurrence [26]. The systematic use of non-invasive tests to monitor graft fibrosis will be of particular interest with a view to confirming in larger series whether the achievement of SVR is associated with the regression of liver fibrosis in the long term [27].

\subsection{Question 8}

Are there antiviral regimens to be favored, in relation to particular HCV genotypes, for treating HCV recurrence?

\subsubsection{Statement}

8a. Antiviral regimens should be selected according to the HCV genotype. To treat early HCV recurrence, 12 weeks of antiviral treatment based on Sofosbuvir and NS5A inhibitors (with or without Ribavirin) is preferable to Paritaprevir/Ritonavir plus Ombitasvir (with or without Dasabuvir) - unless the patient has severe renal failure - due to the former's milder drug-drug interactions (5, D).

\subsubsection{Comment}

In the early weeks after LT, patients are usually given several different drugs in addition to their immunosuppressive medication, so transplant hepatologists should pay attention to drug-to-drug interactions when deciding to use DAAs [28]. Given the relatively recent introduction of DAAs in clinical practice, further clinically significant drug-drug interactions are likely to be discovered in the near future. An unexpected example that emerged only very recently concerns the onset of symptomatic bradycardia when Amiodarone was co-administered with Sofosbuvir in combination with Daclatasvir, Simeprevir or Ledipasvir [29]. As for the duration of antiviral treatments, a short course of antiviral therapy should be preferred to optimize patient adherence [30].

\subsection{Question 9}

Should Ribavirin always be maintained?

\subsubsection{Statement}

9a. The benefit of using Ribavirin in HCV-positive liver recipients was not clearly demonstrated in randomized trials. Data deriving from studies conducted on immune-competent patients indicate that, when treating HCV genotype 1 and 4 infections with 12-week antiviral regimens, Ribavirin could be maintained if the treatment involves Sofosbuvir plus Ledipasvir and Daclatasvir. Combinations of Paritaprevir/Ritonavir plus Ombitasvir and Dasabuvir, or Sofosbuvir plus Simeprevir for genotype $1 \mathrm{~b}$, can be used without Ribavirin, but these combinations are responsible for important drug-drug interactions. A combination of Sofosbuvir plus Daclatasvir can be used without Ribavirin to treat HCV genotype 2 for 12 weeks. Ribavirin should be maintained when treating patients with genotype 3 with advanced liver disease. A prolonged 24-week treatment with Ribavirin should be considered for patients who failed previous DAA regimens (1c, B).

9b. A longer (24-week) antiviral treatment should be preferred for treating genotypes 1, 3 and 4 in patients in whom Ribavirin is contraindicated or poorly tolerated $(4, \mathrm{~B})$.

Renal impairment is a common complication of treatment with calcineurin inhibitors, which are used as the backbone immunosuppressant in LT patients. Ribavirin is a synthetic nucleoside analog metabolized by the liver and excreted by the kidney, so higher serum levels of Ribavirin and its metabolites are seen in patients with renal impairment. LT patients tend to have more side effects, particularly hemolytic anemia, which in many cases necessitate dose reductions or discontinuation of this drug. Antiviral treatment schedules without Ribavirin with a demonstrated efficacy against recurrent HCV infection are consequently needed. For the purpose of treating HCV recurrence, the optimal duration of antiviral treatments and the need for Ribavirin are still controversial issues because no studies have been performed to answer the question of whether or not Ribavirin is necessary, particularly in 12-week antiviral regimens and in cases of non-genotype $2 \mathrm{HCV}$ infection. It is largely accepted, and recommended in current clinical guidelines, that Ribavirin should be used in 12-week treatment schemes, or patients should be treated without Ribavirin for 24 weeks [31]. In kidney transplant recipients with F0-F2 METAVIR chronic hepatitis $\mathrm{C}$, the combination of Sofosbuvir plus Ledipasvir without Ribavirin has been associated with very high SVR12 rates, suggesting that Ribavirin may not be essential in LT recipients with mild disease [32].

\subsection{Question 10}

How should antiviral treatment efficacy and safety be monitored?

\subsubsection{Statement}

10a. The efficacy of antiviral treatment is judged mainly on the grounds of a serum HCV-RNA measurement (using high-sensitivity real-time PCR) obtained 12 weeks after stopping the treatment (1a, A).

10b. Since HCV protease inhibitors have important drug-drug interactions with calcineurin inhibitors, serum levels of immunosuppressive drugs should be checked frequently. Serum levels of hemoglobin, creatinine clearance, and liver function tests should 
be considered the mainstay for monitoring the safety of antiviral treatment (3a, C).

\subsubsection{Comment}

In the less recent clinical trials, a SVR with interferon-based regimens was defined as HCV-RNA serum levels below a designated threshold 24 weeks after stopping the therapy (SVR24). More recently, the regulatory authorities changed this definition of a SVR to mean the absence of HCV-RNA in the serum 12 weeks after stopping the treatment (SVR12), considered a valid marker of a patient cured of any HCV infection. The concordance between SVR12 and SVR24 was recently assessed adopting new DAA regimens [33].

The safety and efficacy profiles of the new DAA regimens were investigated in patients with HCV recurrence, including those with fibrosing cholestatic hepatitis [9,14,34-36]. Although DAAs are generally considered safe in such patients, who are difficult to treat, serum levels of immunosuppressants and hemoglobin (especially if a regimen includes Ribavirin), creatinine clearance and liver function should be checked routinely throughout the treatment and monitored closely afterwards because the achievement of a SVR has been associated with an accelerated liver metabolism of immunosuppressants, leading to the risk of their serum levels dropping.

\subsection{Question 11}

Which antiviral regimens should be adopted in cases of previous treatment failure?

\subsubsection{Statement}

11a. A 24-week retreatment with combinations of Sofosbuvir plus Ribavirin and an NS3 (where NS5A inhibitors have failed), or an NS5A inhibitor (where NS3 inhibitors have failed) seems to be a reasonable rescue strategy if antiviral retreatment cannot be deferred (3b, B).

11b. A retreatment strategy guided by genetic analyses of drug resistance can be proposed for patients with less advanced liver disease in whom combinations based on polymerase plus NS3 inhibitors have failed. There is a stronger recommendation for the performance of genetic analyses of resistance before retreatment in patients in whom a combination based on polymerase plus NS5A inhibitors has failed (5, D).

\subsubsection{Comment}

The treatment of HCV infection with DAAs is associated with high rates of SVR. The factors associated with any failures of this treatment include advanced liver fibrosis, previous failure to respond to DAAs, higher baseline viral loads and the presence of viral particles with resistance-associated substitutions (RAS). These RAS can occur in different genotypes and subtypes if the NS3, NS5A and NS5B regions of HCV, either at the baseline or (more often) as a result of the failure of DAA treatment. Genetic testing is becoming increasingly common nowadays for patients unresponsive to different combinations of DAAs. One study was conducted on 14 patients infected with HCV genotype 1 failing to respond to Sofosbuvir plus Ribavirin for 24 weeks, who were retreated with Sofosbuvir plus Ledipasvir for 12 weeks, and all patients achieved a SVR [37]. This cohort included seven patients with advanced fibrosis/cirrhosis. The S282T variant was transiently detectable in one patient after initial therapy with Sofosbuvir and Ribavirin was stopped, while wild-type NS5B polymerase sequences were observed in all the other patients [37]. In another study, patients infected mainly with HCV genotype 3 who achieved no SVR after 12-24 weeks of Sofosbuvir plus Ribavirin were retreated with either PEGylated interferon with Ribavirin and Sofosbuvir for 12 weeks, or with Sofosbuvir plus Ribavirin for 24 weeks. An interim analysis conducted as part of this study showed that the conventional triple therapy was very effective (SVR12 92\%), while the SVR rate after another course of the Sofosbuvir plus Ribavirin combination therapy was only 63\% [38]. One patient infected with HCV genotype 1 not responding to Sofosbuvir plus Ledipasvir after 8 weeks was successfully retreated using the same regimen with the addition of Ribavirin for 24 weeks, despite the presence of highly resistant NS5A RAVs (per esteso prima?) and a S282T variant responsible for a marked resistance to Sofosbuvir [39]. On the other hand, 41 patients in whom 8-12 weeks of Sofosbuvir plus Ledipasvir, with or without Ribavirin, had failed were given salvage treatment with Sofosbuvir plus Ledipasvir for 24 weeks, achieving an overall SVR rate of $71 \%$. In the subgroup of 11 patients unresponsive to 12 weeks of the previous treatment, only 5 (45\%) achieved a SVR, and the presence of NS5A RAVs was associated with treatment failure [39]. Further studies are ongoing to investigate salvage therapies in patients not responding to DAAs. For the time being, the guidelines recommend waiting to see the results of ongoing clinical studies before retreating patients without severe liver disease, or considering their retreatment with a different class of DAAs, and checking their genetic analyses of resistance, particularly for patients urgently needing retreatment [7].

\section{Prophylaxis for HBV recurrence after liver transplantation}

Remarkable improvements have been made in the outcome of patients transplanted for hepatitis B virus (HBV) in the past two decades. Using standard therapies, the vast majority of transplant recipients are protected against recurrent HBV infection after liver transplantation, and patients transplanted for HBV have a 5-year survival in excess of $80 \%$, which is currently the benchmark for LT performance in adults [40]. These impressive results reflect the success of prophylactic therapies in preventing graft reinfection as well as the greater efficacy of antiviral drugs for managing recurrent post-LT HBV disease. It is crucial to consider the risk of HBV recurrence in order to plan effective strategies against $\mathrm{HBV}$ reactivation post-LT. Risk factors associated with high rates of HBV reactivation are a high viral load prior to the transplant, $\mathrm{HBeAg}$ positivity, HIV co-infection, non-compliance, HCC at the time of the transplant, and drug resistance $[41,42]$. Hepatitis B immunoglobulins (HBIG) have been the core component of HBV prophylaxis since the landmark study by Samuel et al. in 1991, who showed that HBV recurrence could be prevented by the long-term administration of HBIG in $80 \%$ of non-viremic transplant patients $[43,44]$. When the first oral antiviral drug against HBV (Lamivudine) became available, antiviral monotherapy was attempted as an alternative, but failed to maintain results on a par with those achieved by HBIG in terms of HBV recurrence. Thereafter, long-term HBIG and nucleos(t)ide analogues (NAs) became the standard prophylaxis for all patients, whatever their replication status at the time of transplantation [45-47]. High doses of intravenous (iv.) HBIG were often used initially, making this prophylaxis expensive, but the more recent adoption of lower doses of HBIG combined with NA(s) has made the treatment just as effective and less costly, particularly when long-term prophylaxis with intramuscular (im.) or subcutaneous (sc.) low-dose HBIG is combined with more potent third-generation NAs (Entecavir, Tenofovir) [48]. Although combination prophylaxis has proved very successful, some limitations of HBIG are recognized. It requires parenteral administration, for instance, which is inconvenient for patients and care providers. But the feasibility of HBIG-free strategies is still debated, either because of the particular characteristics of large published cohort, or because of different definitions of HBV recurrence (the historical persistence or reappearance of HbsAg vis-à-vis the reappearance of HBV-DNA 
despite antiviral treatment), and the potential residual risk of HCC in immunocompromised patients after the reappearance of HBsAg. Some authors suggested in the past that it might be safe to interrupt HBIG after LT in selected patients (those who undergo LT with undetectable HBV-DNA) [51], and others more recently proposed completely withdrawing HBV prophylaxis after at least 6 years of post-LT follow-up in recipients with undetectable serum and total intrahepatic HBV-DNA, and covalently closed circular DNA at the time of their transplant [52].

\subsection{Question 12}

Which is the best prophylactic regimen for preventing HBV recurrence after $\mathrm{LT}$ in $\mathrm{HBV}+$ recipients?

\subsubsection{Statement}

12a. Nowadays, the best prophylactic regimen - in terms of safety and efficacy - is a combination of third-generation NAs (Entecavir, Tenofovir) plus low-dose HBIG. All transplant centers in Italy adopt this approach in patients transplanted for HBV-related liver disease (5, B).

\subsubsection{Comment}

Recent data emerging from a retrospective study conducted in Far East Countries indicate that monotherapy with thirdgeneration NAs (Entecavir) is effective in preventing recurrent HBV infection after LT in $\mathrm{HBeAg}+$ recipients (the rate of recurrence was $4-5 \%$ ) $[49,50]$. These findings suggest that the current use made of third-generation NAs can minimize the need for HBIG and prove more cost-effective in the long term, but prospective data from dedicated clinical trials will be needed to enable an informed decision on whether to adopt a regimen with third-generation NAs alone (and no HBIG).

\subsection{Question 13}

What is the recommended dose of iv. HBIG to administer perioperatively after LT (in the anhepatic phase and 1st postoperative week)?

\subsubsection{Statement}

13a. The most widely adopted regimen involves the use of $10.000 \mathrm{IU}$ iv. in the anhepatic phase, then $5.000 \mathrm{IU}$ iv. daily until day 7 after LT. Some Italian centers are currently using smaller amounts of HBIG in this perioperative phase, however, with unchanged longterm results, in terms of efficacy $(\mathbf{5}, \mathbf{B})$.

\subsubsection{Comment}

The high iv. dosages of HBIG still used in the perioperative phase at many centers probably come from the time when HBIG were used without NAs, or combined with first-generation NAs (Lamivudine) [51,52]. Now that third-generation NAs (Entecavir, Tenofovir) have become available, lower doses of HBIG can be used in the perioperative phase too. There has been encouraging recent evidence to suggest that administering iv. HBIG based not on preset dosage regimens, but on HBsAg quantification during the initial post-operative clinical course, can maintain a protective anti-HBs titer while saving on the total amount of HBIG required.

\subsection{Question 14}

What is the recommended long-term prophylaxis regimen, in terms of route and timing of HBIG administration?

\subsubsection{Statement}

14a. Lifelong combined NAs + HBIG prophylaxis is administered to prevent HBV recurrence after LT. The currently adopted regimen for this prophylaxis combines third-generation NAs (Entecavir, Tenofovir) with low-dose parenteral HBIG. The im. and sc. routes of HBIG administration have proved equally effective in providing protection, and they can be used starting from shortly after the LT procedure. For long-term protection, the anti-HBs titer should be kept at around $100 \mathrm{IU}(5, \mathrm{~B})$.

\subsubsection{Comment}

Lifelong prophylaxis with NAs and HBIG to maintain an anti-HBs titer of around $100 \mathrm{IU}$ is currently managed using two different approaches. Some centers deliver it on demand (the amount and timing of HBIG administrations being based on anti-HB titers), while others prefer a fixed schedule (usually 2000 IU a month, irrespective of the patient's anti-HBs titer). The two approaches seem to assure the same clinical efficacy and safety, but their costeffectiveness is unknown and studies are needed to clarify this issue.

\subsection{Question 15}

What is the recommended long-term prophylaxis regimen for preventing HBV reactivation after LT in recipients of anti-HBc+ grafts?

\subsubsection{Statement}

15a. Long-term mono-prophylaxis with NAs (Lamivudine) is mandatory in recipients of anti-HBc+ grafts to prevent HBV reactivation after LT. There is evidence from several sources to suggest that HBIG is not helpful in this clinical setting, so they should not be used $(5, B)$.

\subsubsection{Comment}

Recipients of anti-HBc+ grafts are usually given lifelong monoprophylaxis with NAs (Lamivudine) [53-55]. It has been suggested, however, that this prophylaxis might be withdrawn a few years after LT in certain subgroups of patients (depending on their serological HBV status pre-transplant, i.e. patients with an anti$\mathrm{HBcAb}+/ \mathrm{anti}-\mathrm{HBsAb}+$ profile). A dedicated controlled study will be needed before this approach can be translated into clinical practice.

\section{Indications for liver transplantation for alcoholic liver disease}

Alcoholic liver disease is the second most common indication for LT, accounting for approximately $20 \%$ of all primary transplants in Italy, $40 \%$ in Europe and about 25\% in the United States. Although outcomes of LT for alcohol-related liver disease are comparable with those of LT for other etiologies, transplant hepatologists and clinicians have to face several challenging issues when considering patients with alcoholic liver disease for LT. It is hard to say whether the length of the period of pre-LT abstinence has a specific role in predicting post-LT abstinence, or whether it is more useful to identify patients whose condition will improve with abstinence, and who will survive without LT. Most transplant programs demand 6 months of abstinence before considering a patient suitable for LT, but the patient's psycho-social assessment seems more important for identifying potential risk factors of alcohol relapse and establishing the real likelihood of long-term abstinence after LT. Acute alcoholic hepatitis is considered a contraindication for LT at most transplant centers, though early LT in selected patients with a first episode of severe alcoholic hepatitis unresponsive to steroid 
therapy has been shown to improve survival. The LT option should therefore be explored within strict criteria in this setting.

\subsection{Question 16}

Is a 6-month period of abstinence required before a patient can be considered eligible for liver transplantation?

\subsubsection{Statement}

16a. Patients with alcohol-related cirrhosis (with or without HCC):

- the six-month period of abstinence is an arbitrary definition, but remains a valid criterion for this subset of patients, mainly because it enables the liver's potential for functional improvement to be assessed $(\mathbf{4}, \mathrm{C})$;

- all patients should undergo a multidisciplinary assessment with an addiction specialist, psychiatrist, psychologist and/or social worker to identify risk factors for non-adherence before and after LT $(5, D)$;

- if patients' liver disease has not stabilized within 6 months, they can be considered for LT;

- if their liver function deteriorates, LT is indicated even without 6 months of abstinence (5, D).

16b. Patients with alcohol-related cirrhosis $(+/-\mathrm{HCC})$ with a life expectancy of less than 6 months:

- all patients should undergo a multidisciplinary assessment with an addiction specialist, psychiatrist, psychologist and/or social worker to identify risk factors for non-adherence before and after LT (5, D).

\subsubsection{Comment}

The rationale behind this strategy is to consider the period of abstinence before LT as a tool for avoiding LT in patients whose liver function will improve with abstinence alone, and identifying patients at higher risk of post-LT alcohol relapse. This approach can be applied to patients with a life expectancy beyond 6 months. For patients with a shorter life expectancy, the 6-month period of abstinence is not mandatory, and other parameters should be considered to judge the risk of post-LT alcohol relapse. Some of the most important are family and social support, prior psychiatric comorbidities, unsuccessful attempts at rehabilitation, and a family history of alcoholism [56].

\subsection{Question 17}

How should abstinence be monitored while patients are on the waiting list?

\subsubsection{Statement}

17a. Carbohydrate deficient transferrin (CDT) assays are not useful for assessing alcohol consumption during the required period of abstinence $(\mathbf{4}, \mathbf{C})$.

17b. Where available, urinary ethyl glucuronide (uEtG) assay should always be performed in stable cirrhotic patients $(4, \mathbf{C})$.

17c. The frequency of monitoring by the addiction specialist, psychologist and/or psychiatrist can vary, at the discretion of the various specialists $(\mathbf{5}, \mathbf{D})$.

\subsubsection{Comment}

Transplant hepatologists and clinicians involved in the care of patients with alcoholic liver disease should focus on detecting any form of alcohol use during the assessment process or while patients are on the waiting list for LT. Among the screening tools available, CDT assay is of limited use because it is unable to identify the intake of small quantities of alcohol, and serum levels are often unpredictable because of the interference of hyperbilirubinemia [57]. Instead, uEtG assay is considered a reliable and useful screening tool in the LT setting $[58,59]$ as it is detectable even after the consumption of very small amounts of ethanol $(<10 \mathrm{~g})$, and for up to $80 \mathrm{~h}$ after the last drink was ingested [60].

\subsection{Question 18}

Is a patient with polysubstance abuse and end-stage liver disease a potential candidate for liver transplantation?

\subsubsection{Statement}

18a. Smoking and the use of cannabinoids and methadone do not make LT absolutely contraindicated, but patients with polysubstance abuse need to be adequately assessed by a multidisciplinary team (addiction specialist, psychiatrist, psychologist, social worker) to examine how they used these substances and their potential dependence (2b, B).

$\mathbf{1 8 b}$. The active use of opioids, cocaine and synthetic drugs is an absolute contraindication for LT (2b, B).

\subsubsection{Comment}

Although smoking is not considered an absolute contraindication for LT, it should be mandatory for patients to stop smoking before LT because it is associated with higher post-transplant morbidity and mortality rates.

\subsection{Question 19}

What is appropriate for the management of alcohol and/or other substance use/abuse before liver transplantation?

\subsubsection{Statement}

19a. Anti-craving therapy options are limited before LT, but gamma-hydroxybutyric acid (GHB), Baclofen and Topiramate can be used in cirrhotic patients $(3, \mathrm{C})$.

19b. Individual or group-based psychological support should be offered to these patients, including specialist help with the management of alcohol-related problems or psychiatric disorders, and self-help groups $(\mathbf{4}, \mathrm{C})$.

19c. Hepatological outpatient visits should be scheduled according to the severity of a patient's liver disease:

- at least every 3 months for stable disease;

- at least once a month for unstable/severe liver disease $(4, C)$.

\subsubsection{Comment}

New studies are needed to improve our knowledge in this field, to define a follow-up protocol that should be adopted by all transplant centers in Italy, and to nurture collaborations with services outside the hospital, including self-help groups, mental health services, and drug addiction services.

\subsection{Question 20}

Is acute alcoholic hepatitis, as a first episode of decompensation in patients with chronic liver disease, an indication for liver transplantation?

\subsubsection{Statement}

20a. Patients with acute alcoholic hepatitis, as a first episode of decompensation in chronic liver disease, and not responding 
to steroid therapy can only be considered for LT if the following conditions are met $(\mathbf{2 b}, \mathbf{B})$ :

- total consensus of the paramedical and medical staff;

- no comorbidities;

- social integration;

- supportive family members;

- psychiatric assessment and addiction profile.

Non-responders to steroid therapy are identified on the grounds of a Lille score $\geq 0.45$ or worsening liver function by day 7 (1), $\mathbf{A})$.

Patients unsuitable for steroid therapy, with a MELD score $>30$, can only be considered for LT if they meet the above criteria (2b, B).

\subsubsection{Comment}

It has been demonstrated that early LT for severe acute alcoholic hepatitis only has potential as a therapeutic option for patients not responding to steroid therapy if specific, strict selection criteria are met [61]. Sequential infection screening before steroid therapy is an efficient way to identify patients at high risk of developing infections [62]. Antibiotic therapy can be administered as an adjuvant to steroids in the setting of severe acute alcoholic hepatitis [63]. Studies are warranted on the usefulness of fungal prophylaxis before LT in the light of reported deaths due to aspergillosis. Further multicenter studies are needed to explore this controversial issue properly.

20b. The diagnosis of acute alcoholic hepatitis is based mainly on a clinical examination, but a transjugular liver biopsy should be obtained, where the option is available, at highly-experienced centers $(\mathbf{4}, \mathbf{C})$.

A histological diagnosis is not required for steroid therapy $(\mathbf{4}, \mathbf{C})$.

Any presence of infections and/or sepsis should always be ruled out before starting steroid therapy (2b, B).

Steroid therapy should be administered to patients with a discriminant factor (DF) $\geq 32$ and should be based on methylprednisolone $40 \mathrm{mg} /$ day (2b, B).

When patients are potential candidates for LT, steroid therapy should always be provided at a liver transplant center $(\mathbf{5}, \mathbf{D})$.

\subsubsection{Comment}

Although acute alcoholic hepatitis is considered a clinical syndrome and its diagnosis is often based on clinical and biochemical findings, the role of liver biopsy in establishing a diagnosis of alcoholic hepatitis remains controversial. This is due to the higher risk of bleeding in such patients as they often present with coagulopathy and thrombocytopenia. Transjugular liver biopsy is consequently preferable, if available, since the procedure is safe even in patients with a low platelet count [64]. In our opinion, a histological diagnosis is not a prerequisite for steroid therapy at experienced centers, though this attitude is not perfectly in line with the EASL Clinical Practice Guidelines on Liver Transplantation [65].

\subsection{Question 21}

What is the correct follow-up after LT?

\subsubsection{Statement}

21a. A post-LT assessment by the addiction specialist, psychologist and/or psychiatrist (depending on availability at the transplant center) is strongly recommended, at intervals suited to the patient's characteristics and transplant center variables. The high risk of de novo tumors in these patients warrants a strict, regular follow-up $(5, \mathrm{C})$.

21b. This is mandatory for patients transplanted with potential risk factors for post-LT alcohol relapse $(5, \mathbf{C})$. 21c. Anti-craving therapy is feasible in the post-LT setting (5, D).

\subsubsection{Comment}

An adequate post-LT follow-up is crucial in order to contain the rate of alcohol relapse. This should include assessment by the addiction specialist, psychologist and/or psychiatrist, and should focus on patients with specific pre-LT risk factors for post-LT alcohol relapse. Pharmacological therapy can be considered after LT.

\subsection{Question 22}

Is graft dysfunction due to alcohol relapse a potential indication for liver re-transplantation?

\subsubsection{Statement}

22a. Graft dysfunction due to alcohol relapse is considered a contraindication for liver re-transplantation $(\mathbf{2 b}, \mathbf{B})$.

\subsubsection{Comment}

To date, only a few cases have been reported of liver retransplantation for graft dysfunction due to alcohol relapse.

\section{Immunosuppressive therapy}

As a general concept, a transplanted liver is better "tolerated" by the recipient than other organs, so liver recipients are maintained on lower levels of immunosuppression than other organ transplant recipients. In a small percentage of selected LT recipients, the allograft may even survive long after immunosuppressant therapy has been withdrawn. Rejection of transplanted livers is nonetheless a diagnostic challenge and there is an ongoing search for reliable and readily-available early markers of this condition. Immunosuppression protocols used at liver transplant centers differ widely and there is a general tendency for individual recipient-tailored immunosuppression, generally based on different indications for LT and comorbidities. Concerning the indications for LT, HCC and recipient serum HCV RNA positivity (though the latter will become rare in future due to pre-LT pharmacological HCV clearance) are two conditions that could, in principle, influence immunosuppressive strategies. As for pre- and/or post-transplant comorbidities, renal dysfunction, metabolic syndrome (or single components thereof, such as obesity, diabetes, hypertension and dyslipidemia) and postLT de novo neoplasms could also dictate the choice of different immunosuppression protocols.

\subsection{Question 23}

What are the suggested regimens for administering steroids by recipient pre-transplant diagnosis and post-transplant follow-up?

\subsubsection{Statement}

23a. Based on the available evidence, steroid-free immunosuppression after LT should be considered in all LT recipients except for patients transplanted for autoimmune hepatitis, primary biliary cholangitis, or primary sclerosing cholangitis (1a, $\mathbf{A})$.

\subsubsection{Comment}

Except in patients with autoimmune hepatitis, primary biliary cholangitis or primary sclerosing cholangitis, the routine use of steroids in the first 6 months after LT does not improve graft or patient survival $[66,67]$, and it is associated with a higher incidence of CMV infection and more severe HCV recurrences after LT [68-70]. 


\subsection{Question 24}

When should a calcineurin-sparing/withdrawal strategy be adopted in the case of renal dysfunction, and how should this strategy be implemented?

\subsubsection{Statement}

24a. In patients with pre-LT renal dysfunction, it is advisable to delay the introduction of calcineurin inhibitors (CNIs), or to reduce the dose of CNIs and add mycophenolic acid (MMF) or enteric-coated mycophenolate sodium (EC-MPS), and/or IL-2 receptor antibodies (1), $\mathbf{A})$.

24b. In patients who develop renal failure after LT, a combination of MMF or EC-MPS and reduced-dose Tacrolimus (TAC) is recommended (1a, $\mathbf{A})$.

24c. A valid alternative is a combination of Everolimus (EVE) with reduced-dose TAC (1), B).

\subsubsection{Comment}

In patients with pre-LT renal dysfunction, the delayed introduction or reduced doses of CNIs under the protection of MMF and/or IL-2 receptor antibodies is associated with an improvement in renal function in the short and mid-term, with no increase in the risk of liver rejection [71]. In patients who develop renal failure after LT a combination of MMF and reduced-dose TAC has proved a safe and effective approach to ameliorating renal dysfunction [72-75]. Gastrointestinal symptoms in LT recipients are more common with MMF treatment than with EC-MPS, and conversion from MMF to EC-MPS leads to symptom improvement [76-80].

Compared with standard therapy, a combination of EVE with reduced-dose TAC as a de novo (within 1 month of the transplant) or as a conversion immunosuppressive regimen is associated with an equally effective immunosuppression and no renal toxicity. The risks of adverse wound healing events with EVE is associated with the administration of a loading dose. Using lower doses of EVE without a loading dose is not associated with any increased risk of wound healing complications. No conclusive data are available on the impact of EVR on wound healing in the event of its de novo use after LT $[81,82]$.

\subsection{Question 25}

What are the immunosuppressive strategies for HCV-RNA+ recipients?

\subsubsection{Statement}

25a. Either TAC-based or cyclosporine (CSA)-based immunosuppression can be used in HCV-infected patients after LT (1a, A).

25b. Steroid-free immunosuppression is safe in $\mathrm{HCV}$-positive LT recipients (1a, B).

\subsubsection{Comment}

Neither the HCV recurrence-related mortality, graft loss and retransplantation rates, nor the incidence of histological HCV recurrence, is associated with the choice of CNIs administered [81]. Steroid-free immunosuppression is safe in HCV-positive LT recipients and does not increase the risk of rejection or of fibrosis progression [82].

\subsubsection{Question 26}

What are the immunosuppressive strategies for patients transplanted for HCC?

\subsection{Statement}

26a. Both CyA and TAC reportedly raise the post-LT risk of HCC recurrence in a dose-dependent manner [4].

26b. There is no strong evidence for the use of immunosuppressive regimens based on mammalian target of rapamycin inhibitors (mTORi) to reduce HCC recurrences (2b, B), but they do seem to reduce the rate of HCC recurrence in low-risk (Milan Criteria in criteria) patients (1a, B).

\subsubsection{Comment}

It is still not clear which immunosuppressive therapy is best to reduce HCC recurrences, despite the anti-proliferative effect of mTORi and the positive effect of CNI weaning [83]. Although there is no strong evidence for the use of mTORi-based immunosuppressive regimens [84], the SILVER study has suggested a lower HCC recurrence rate in low-risk patients (Milan in criteria) [85].

\subsection{Question 27}

Which immunosuppressive strategies are appropriate when a de novo neoplasm develops after LT?

\subsubsection{Statement}

27a. The risk of de novo malignancy is $2-4$ times higher in transplant recipients. The types of cancer with the highest standardized incidence ratio after LT related to viral infections originate from the immune system (2b).

27b. The incidence of the overall cancer is lower when mTORi immunosuppression regimens are adopted, but there is no strong evidence to support the use of mTORi to reduce the incidence of

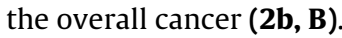

\subsubsection{Comment}

For the time being, there is no strong evidence to indicate which is the best immunosuppressive therapy in cases of de novo neoplasms post-LT $[84,86]$, so minimizing immunosuppression, screening and avoiding risk factors remain the main recommendations for managing the risk of de novo malignancies [87].

\subsection{Question 28}

Which immunosuppressive strategies are appropriate in cases of metabolic syndrome?

\subsubsection{Statement}

28a. Based on the currently available evidence, there is no specific immunosuppressive therapy for managing patients with metabolic syndrome (MS). It is reasonable to consider tailored therapies according to each patient's single MS components, especially in the event of diabetes and hypertension $(\mathbf{2 b}, \mathbf{B})$.

\subsubsection{Comment}

The prevalence of MS is high (38\%-50\%), and rising among post-LT patients, and is associated with a higher likelihood of cardiovascular and cerebrovascular events [88]. Different types of immunosuppression (TAC vs. CsA vs. others) assessed at 1 year, and corticosteroid use in the first 3-6 months were not significantly associated with the subsequent onset of MS $[89,90]$. In the case of diabetes (considered as a separate MS component), only methylprednisolone boluses emerged as independent risk factors for the onset of post-LT diabetes [91]. A meta-analysis of sixteen prospective, randomized comparative studies reported a $13.4 \%$ incidence of new-onset diabetes in patients after solid organ transplantation, with a higher incidence in patients receiving TAC than in those given CsA (16.6\% vs. 9.8\%). This trend was observed across 
renal, liver, heart and lung transplant groups [92]. The association between BMI and/or obesity at 1 year and type of immunosuppression (TAC vs. CsA vs. others) was not found statistically significant [93]. Hypertension occurs with both types of CNI, but more commonly with CsA, compared with which TAC was found associated with a lower incidence of hypertension and cardiovascular disease [89]. Early conversion from CNI therapy to either Sirolimus or Everolimus is associated with higher serum triglyceride and cholesterol levels [94,95].

\subsection{Question 29}

How should acute, late acute, and chronic rejection be diagnosed?

\subsubsection{Statement}

29a. Blood concentrations of immunosuppressive drugs should be assayed as standard procedure $(\mathbf{4}, \mathbf{B})$.

29b. Generic TAC and MMF can be used safely as a conversion from original branded drugs $(4, \mathrm{C})$.

29c. In LT recipients with an unexplained graft dysfunction, a biopsy should be obtained to rule out acute rejection (3, B).

29d. The value of searching for preformed (i.e. pre-LT) donorspecific antibodies (DSAs) in the serum is still controversial because its role in immune-mediated liver damage is not clear $(\mathbf{4}, \mathbf{C})$.

29e. Persistent DSA positivity may be associated with early and severe acute antibody-mediated rejection (ABMR) (2b).

29f. Complement component $4 \mathrm{~d}$ (C4d) staining in liver biopsy should only be used in selected cases when isolated or predominantly ABMR is suspected $(\mathbf{4}, \mathbf{C})$.

\subsubsection{Comment}

The use of generic TAC and MMF has proved safe and costeffective as a strategy for conversion from original branded drugs. Data on the de novo use of generic immunosuppressants after LT are still not conclusive [96-98].

DSAs occur in up to $15 \%$ of LT recipients, but disappear after the procedure in most patients [94,99].

De novo DSAs develop in $8-14 \%$ of LT recipients, depending on how soon after the operation the assay is performed and on the mean fluorescence intensity cut-off used to define DSA positivity. De novo DSA development may be associated with acute antibodymediated rejection $[100,101]$.

DSAs detected in maintenance LT patients are associated with a higher risk of acute rejection and a shorter allograft survival [99,101-103].

C4d staining is not specific for any particular liver graft injury and can only provide additional information to consider in the context of other pathological and clinical features. It may be a marker of isolated ABMR (a rare occurrence in liver grafts), or of antibody-mediated injury associated with acute cellular rejection, or an aspecific finding in other graft diseases (chronic hepatitis, bile duct or vascular injuries). Diffuse C4d positivity as a sign of ABMR in more than $50 \%$ of the vascular compartment [104].

Promising results have emerged from eosinophil counts (which have revealed a strong association with moderate-severe acute cellular rejection), CD4 function tests (used to identify overimmunosuppression, but not under-immunosuppression), and anti-HLA donor-specific antibody assays (to diagnose acute antibody-mediated rejection) [99,105-107].

\subsection{Question 30}

Is there a role for an Italian multicenter clinical trial on drug minimization and/or withdrawal?

\subsubsection{Statement}

30a. There is a role for such trial because the withdrawal of immunosuppression to induce operational tolerance should be managed at liver transplant centers with specific experience of this matter, selecting patients with a long time having elapsed since LT and with a normal liver function $(\mathbf{2 b}, \mathbf{A})$.

\subsubsection{Comment}

Spontaneous operational tolerance (i.e. the complete withdrawal of immunosuppression) is feasible in nearly $20 \%$ of LT patients receiving a whole cadaveric organ, and is associated with a reduction of immunosuppression-related side effects and complications $[108,109]$. The time elapsing after LT is a major factor influencing spontaneous operational tolerance, which is rarely seen in patients transplanted less than 6 years earlier, and more common (79\% of cases) in those transplanted more than 10 years before [110]. To demonstrate the real clinical utility of operational tolerance, large multicenter studies will be needed, using a panel of different biomarkers (including NK cells and their related transcripts, serum levels of hepcidin and ferritin and graft-derived cell-free DNA) is needed to assess immunological suppression adequately, and thus modify immunosuppressive treatments according to patients' needs [111-113].

\section{Conclusion}

The aim of this position paper prepared by the AISF's Permanent Committee on Liver Transplantation is to provide recommendations for orienting the clinical activity of transplant hepatologists managing patients on the waiting list for LT and after their transplantation in real-life clinical practice. The recommendations concern 5 of the most common issues in liver transplantation, including how to manage patients with hepatitis $C$, hepatitis $B$, and alcoholic liver disease, and immunosuppression.

It is well known that HCV-related liver disease is the most common indication for LT. HCV recurrence after LT was considered inevitable until recently, but the newly-developed, very effective and well tolerated antiviral drugs for treating patients before and after LT have completely changed the scenario of anti-HCV therapy in this setting.

HBV-related liver disease is a standard indication for LT. With current antiviral therapy before the transplant and HBV prophylaxis afterwards, the HBsAg recurrence rate in the long-term follow-up is lower than $10 \%$ of cases, and the 5 -year survival rate exceeds $80 \%$ of cases. The prophylaxis adopted at different liver transplant centers varies considerably, however, and there is still much debate as to which drugs or drug combinations, routes of administration, and protective anti-HBs titers should be used, and for how long this prophylaxis is necessary.

Alcoholic liver disease represents the second most common indication for LT and still poses a challenge for transplant hepatologists. Most (if not all) liver transplant centers in Italy demand a 6-month period of abstinence before adding patients with alcoholic liver disease to the waiting list for LT. A pre-transplant psycho-social assessments to identify potential risk factors of alcohol relapse after LT is not routinely performed at all centers. Acute alcoholic hepatitis, which may develop on top of chronic liver disease, is still considered a contraindication for LT at most transplant centers, even though early LT in carefully selected patients with a first episode of severe alcoholic hepatitis failing to respond to steroid therapy has been shown to improve survival.

Finally, there is the general, transversal topic of immunosuppression and the diagnosis and treatment of immune-mediated disorders following LT. Despite nearly 30 years of experience with LT in Italy, approaches differ widely from one liver transplant cen- 
ter to another. Some centers adopt the same immunosuppressive regimen whatever the etiology of liver disease, while others follow different regimens for patients transplanted for viral hepatitis or hepatocellular carcinoma. As concerns any pre-transplant comorbities, such as renal dysfunction, metabolic syndrome or single components of MS, such as obesity, diabetes, hypertension and dyslipidemia, there is no homogeneity in the immunosuppression protocols adopted post-LT. The same appears to apply in the case of de novo tumors developing after the transplant, and the diagnosis and treatment of liver dysfunctions occurring due to immunemediated damage is even more complicated.

As mentioned earlier, this position paper has been prepared to assist transplant hepatologists assessing candidates for liver transplantation, and to help them manage transplanted patients correctly. We hope it will also be useful for the purpose of broadening the discussion to other healthcare providers potentially interested and/or involved in the continuously-evolving process of organ donation and liver transplantation.

\section{Conflict of interest}

None declared.

\section{Appendix A.}

Group 1 - New treatment for HCV in patients on the waiting list for liver transplantation

Coordinator: Luca Saverio Belli.

Working group: Paola Carrai, Francesca Donato, Alfonso LanzaGaleota, Silvia Martini, Luisa Pasulo, Francesca Ponziani, Francesco Paolo Russo.

Group 2 - Antiviral treatment in patients with $\mathrm{HCV}$ recurrence after liver transplantation

Coordinator: Pierluigi Toniutto.

Working group: Carmine Coppola, Paolo Forte, Chiara Mazzarelli, Manuela Merli, Marzia Montalbano, Francesco Paolo Picciotto, Maria Rendina, Alberto Zanetto.

Group 3 - Prophylaxis for HBV recurrence after liver transplantation

Coordinator: Riccardo Volpes.

Working group: Paolo Angeli, Francesco Foschi, Matteo Manini, Alfredo Marzano, Giovanni Perricone, Paolo Pianta, Mariarosa Tamè.

Group 4 - Indications for liver transplantation for alcoholic liver disease

Coordinator: Patrizia Burra.

Working group: Nicola De Maria, Marco Domenicali, Giacomo Germani, Antonio Ottobrelli, Antonio Picciotto, Laura Ponti, Giovanni Vizzini.

Group 5 - Immunosuppressive therapy

Coordinator: Stefano Ginanni Corradini.

Working group: Sherrie Bhoori, Flaminia Ferri, Giovanna Gaffuri, Ilaria Lenci, Laura Mameli, Maria Cristina Morelli, Maria Rosaria Piras.

\section{References}

[1] Available from: http://www.cebm.net/oxford-centre-evidence-basedmedicine-levels-evidence-march-2009. 2009. [Cited 26 September 2016].

[2] Available from: http://www.eltr.org/. 2016. [Cited 26 September 2016].

[3] Available from: https://www.unos.org/. 2016 [Cited 26 September]

[4] Garcia-Retortillo M, Forns X, Feliu A, et al. Hepatitis C virus kinetics during and immediately after liver transplantation. Hepatology 2002;35:680-7.

[5] Gane E. The natural history and outcome of liver transplantation in hepatitis C virus-infected recipients. Liver Transplantation 2003;9:S28-34

[6] Forman LM, Lewis JD, Berlin JA, et al. The association between hepatitis C infection and survival after orthotopic liver transplantation. Gastroenterology 2002:122:889-96.

[7] European Association For Study of L. EASL. Recommendations on Treatment of Hepatitis C 2015. Journal of Hepatology 2015;63:199-236.
[8] Belli LS, Berenguer M, Cortesi PA, et al. Delisting of liver transplant candidates with chronic hepatitis C after viral eradication: a European study. Journal of Hepatology 2016;65:524-31.

[9] Charlton M, Everson GT, Flamm SL, et al. Ledipasvir and sofosbuvir plus ribavirin for treatment of HCV infection in patients with advanced liver disease. Gastroenterology 2015;149:649-59.

[10] Foster GR, Irving WL, Cheung MC, et al. Impact of direct acting antiviral therapy in patients with chronic hepatitis $\mathrm{C}$ and decompensated cirrhosis. Journal of Hepatology 2016;64:1224-31.

[11] Manns M, Samuel D, Gane EJ, et al. Ledipasvir and sofosbuvir plus ribavirin in patients with genotype 1 or 4 hepatitis $C$ virus infection and advanced liver disease: a multicentre, open-label, randomised, phase 2 trial. The Lancet Infectious Diseases 2016;16:685-97.

[12] Aqel BA, Pungpapong S, Leise M, et al. Multicenter experience using simeprevir and sofosbuvir with or without ribavirin to treat hepatitis $C$ genotype 1 in patients with cirrhosis. Hepatology 2015;62:1004-12.

[13] Curry MP, O’Leary JG, Bzowej N, et al. Sofosbuvir and velpatasvir for HCV in patients with decompensated cirrhosis. The New England Journal of Medicine 2015;373:2618-28

[14] Poordad F, Schiff ER, Vierling JM, et al. Daclatasvir with sofosbuvir and ribavirin for hepatitis $C$ virus infection with advanced cirrhosis or post-liver transplantation recurrence. Hepatology 2016;63:1493-505.

[15] Cheung MC, Walker AJ, Hudson BE, et al. Outcomes after successful direct-acting antiviral therapy for patients with chronic hepatitis $\mathrm{C}$ and decompensated cirrhosis. Journal of Hepatology 2016:65:741-7.

[16] Curry MP, Forns X, Chung RT, et al. Sofosbuvir and ribavirin prevent recurrence of HCV infection after liver transplantation: an open-label study. Gastroenterology 2015;148:100-7, e1

[17] Reig M, Marino Z, Perello C, et al. Unexpected high rate of early tumor recurrence in patients with HCV-related HCC undergoing interferon-free therapy. Journal of Hepatology 2016;65:719-26.

[18] ANRS collaborative study group on hepatocellular carcinoma Lack of evidence of an effect of direct-acting antivirals on the recurrence of hepatocellular carcinoma: data from three ANRS cohorts. Journal of Hepatology 2016;65:734-40.

[19] Donato MF, Monico S, Malinverno F, et al. Bridging all oral DAA therapy from wait time to post-liver transplant to improve HCV eradication? Liver International 2015;35:1-4.

[20] Forns X, Charlton M, Denning J, et al. Sofosbuvir compassionate use program for patients with severe recurrent hepatitis $C$ after liver transplantation. Hepatology 2015;61:1485-94.

[21] Vigano M, Colombo M. Extrahepatic manifestations of hepatitis C virus. Gastroenterology Clinics of North America 2015;44:775-91.

[22] Makara M, Sulyok M, Csacsovszki O, et al. Successful treatment of HCV-associated cryoglobulinemia with ombitasvir/paritaprevir/ritonavir, dasabuvir and ribavirin: a case report. Journal of Clinical Virology 2015;72:66-8.

[23] Carrion JA, Navasa M, Garcia-Retortillo M, et al. Efficacy of antiviral therapy on hepatitis $C$ recurrence after liver transplantation: a randomized controlled study. Gastroenterology 2007:132:1746-56.

[24] Bedossa P, Poynard T. An algorithm for the grading of activity in chronic hepatitis C. The METAVIR Cooperative Study Group. Hepatology 1996;24:289-93.

[25] Gambato M, Crespo G, Torres F et al Simple prediction of long-term clinica outcomes in patients with mild hepatitis c recurrence after liver transplantation. Transplant International 2015;29:698-706.

[26] Bignulin S, Falleti E, Cmet S, et al. Usefulness of acoustic radiation force impulse and fibrotest in liver fibrosis assessment after liver transplant. Annals of Hepatology 2016;15:200-6.

[27] Burra P, De Martin E, Zanetto A, et al. Hepatitis C virus and liver transplantation: where do we stand? Transplant International 2016;29:135-52.

[28] Kwo PY, Badshah MB. New hepatitis C virus therapies: drug classes and metabolism, drug interactions relevant in the transplant settings, drug options in decompensated cirrhosis, and drug options in endstage renal disease. Current Opinion in Organ Transplantation 2015;20: 235-41.

[29] Brainard DM, McHutchison JG. Bradyarrhythmias associated with sofosbuvir treatment. The New England Journal of Medicine 2015:373:1888.

[30] Petersen T, Townsend K, Gordon LA, et al. High adherence to all-oral directly acting antiviral HCV therapy among an inner-city patient population in a phase 2a study. Hepatology International 2016;10:310-9.

[31] EASL recommendations on treatment of hepatitis C. Journal of Hepatology 2015;63:199-236.

[32] Colombo M, Aghemo A, Liu H, et al. Treatment with ledipasvir-sofosbuvir for 12 or 24 weeks in kidney transplant recipients with chronic hepatitis C Virus genotype 1 or 4 infection: a randomized trial. Annals of Internal Medicine 2016 [Epub ahead of print]

[33] Yoshida EM, Sulkowski MS, Gane EJ, et al. Concordance of sustained virological response 4,12 , and 24 weeks post-treatment with sofosbuvir-containing regimens for hepatitis C virus. Hepatology 2015;61:41-5.

[34] Kwo PY, Mantry PS, Coakley E, et al. An interferon-free antiviral regimen for HCV after liver transplantation. The New England Journal of Medicine 2014;371:2375-82.

[35] Poordad F, Schiff ER, Vierling JM, et al. Daclatasvir with sofosbuvir and ribavirin for HCV infection with advanced cirrhosis or post-liver transplan recurrence. Hepatology 2016;63:1493-505 
[36] Pungpapong S, Aqel B, Leise M, et al. Multicenter experience using simeprevir and sofosbuvir with or without ribavirin to treat hepatitis $C$ genotype 1 after liver transplant. Hepatology 2015;61:1880-6.

[37] Osinusi A, Kohli A, Marti MM, et al. Re-treatment of chronic hepatitis C virus genotype 1 infection after relapse: an open-label pilot study. Annals of Internal Medicine 2014;161:634-8.

[38] Esteban RNL, Lalezari J, Ni L, et al. Successful retreatment with sofosbuvircontaining regimens for HCV genotype 2 or 3 infected patients who failed prior sofosbuvir plus ribavirin therapy. Hepatology 2014;60:S4-5.

[39] Lawitz EFS, Yang J, Pang PS, et al. Retreatment of patients who failed 8 or 12 weeks of ledipasvir/sofosbuvir-based regimens with ledipasvir/sofosbuvir for 24 weeks. Journal of Hepatology 2015;62:S192.

[40] Burra P, Germani G, Adam R, et al. Liver transplantation for HBV-related cirrhosis in Europe: an ELTR study on evolution and outcomes. Journal of Hepatology 2013;58:287-96.

[41] Buti M, Mas A, Prieto M, et al. A randomized study comparing lamivudine monotherapy after a short course of hepatitis B immune globulin (HBIg) and lamivudine with long-term lamivudine plus HBIg in the prevention of hepatitis B virus recurrence after liver transplantation. Journal of Hepatology 2003;38:811-7.

[42] Lenci I, Baiocchi L, Tariciotti L, et al. Complete hepatitis B virus prophylaxis withdrawal in hepatitis B surface antigen-positive liver transplant recipients after longterm minimal immunosuppression. Liver Transplantation 2016;22:1205-13.

[43] Marzano A. The prevention of viral recurrence in the long term. Digestive and Liver Disease 2009;41(Suppl. 2):S195-7.

[44] Samuel D, Bismuth A, Mathieu D, et al. Passive immunoprophylaxis after liver transplantation in HBsAg-positive patients. Lancet 1991;337:813-5.

[45] Angus PW, McCaughan GW, Gane EJ, et al. Combination low-dose hepatitis B immune globulin and lamivudine therapy provides effective prophylaxis against posttransplantation hepatitis B. Liver Transplantation 2000:6:429-33.

[46] Marzano A, Salizzoni M, Debernardi-Venon W, et al. Prevention of hepatitis B virus recurrence after liver transplantation in cirrhotic patients treated with lamivudine and passive immunoprophylaxis. Journal of Hepatology 2001;34:903-10.

[47] Naoumov NV, Lopes AR, Burra P, et al. Randomized trial of lamivudine versus hepatitis B immunoglobulin for long-term prophylaxis of hepatitis B recurrence after liver transplantation. Journal of Hepatology 2001:34:888-94.

[48] Cholongitas E, Papatheodoridis GV. High genetic barrier nucleos(t)ide analogue(s) for prophylaxis from hepatitis B virus recurrence after liver transplantation: a systematic review. American Journal of Transplantation 2013;13:353-62

[49] Fung J, Chan SC, Cheung SC, et al. Oral nucleoside/nucleotide analogs without hepatitis B immune globulin after liver transplantation for hepatitis B. American Journal of Gastroenterology 2013;108:942-8.

[50] Fung J, Lo R, Chan SC, et al. Outcomes including liver histology after liver transplantation for chronic hepatitis B using oral antiviral therapy alone. Liver Transplantation 2015;21:1504-10.

[51] Fernandez I, Loinaz C, Hernandez O, et al. Tenofovir/entecavir monotherapy after hepatitis B immunoglobulin withdrawal is safe and effective in the prevention of hepatitis B in liver transplant recipients. Transplant Infectious Disease 2015:17:695-701.

[52] Terrault N. Prophylaxis in HBV-infected liver transplant patients: end of the HBIG era? The American Journal of Gastroenterology 2013;108:949-51.

[53] Cholongitas E, Papatheodoridis GV, Burroughs AK. Liver grafts from antihepatitis B core positive donors: a systematic review. Journal of Hepatology 2010;52:272-9.

[54] Saab S, Waterman B, Chi AC, et al. Comparison of different immunoprophylaxis regimens after liver transplantation with hepatitis B core antibody-positive donors: a systematic review. Liver Transplantation 2010;16:300-7.

[55] Vizzini G, Gruttadauria S, Volpes R, et al. Lamivudine monoprophylaxis for de novo HBV infection in HBsAg-negative recipients with HBcAb-positive liver grafts. Clinical Transplantation 2011;25:E77-81.

[56] McCallum S, Masterton G. Liver transplantation for alcoholic liver disease: a systematic review of psychosocial selection criteria. Alcohol and Alcoholism 2006;41:358-63.

[57] Piccinelli M, Tessari E, Bortolomasi M, et al. Efficacy of the alcohol use disorders identification test as a screening tool for hazardous alcohol intake and related disorders in primary care: a validity study. BMJ 1997;314:420-4.

[58] Dimartini A, Day N, Dew MA, et al. Alcohol use following liver transplantation: a comparison of follow-up methods. Psychosomatics 2001;42:55-62.

[59] Hietala J, Puukka K, Koivisto H, et al. Serum gamma-glutamyl transferase in alcoholics, moderate drinkers and abstainers: effect on gt reference intervals at population level. Alcohol and Alcoholism 2005;40:511-4.

[60] Nyblom H, Berggren U, Balldin J, et al. High AST/ALT ratio may indicate advanced alcoholic liver disease rather than heavy drinking. Alcohol and Alcoholism 2004;39:336-9.

[61] Mathurin P, Moreno C, Samuel D, et al. Early liver transplantation for severe alcoholic hepatitis. The New England Journal of Medicine 2011;365:1790-800.

[62] Mookerjee RP, Stadlbauer V, Lidder S, et al. Neutrophil dysfunction in alcoholic hepatitis superimposed on cirrhosis is reversible and predicts the outcome. Hepatology 2007;46:831-40.
[63] Louvet A, Wartel F, Castel H, et al. Infection in patients with severe alcoholic hepatitis treated with steroids: early response to therapy is the key factor. Gastroenterology 2009;137:541-8.

[64] Under the Auspices of the Italian Association for the Study of Liver Diseases and the Italian Society of Internal Medicine. Hemostatic balance in patients with liver cirrhosis: report of a consensus conference. Digestive and Liver Disease 2016;48:455-67.

[65] European Association for the Study of the Liver. EASL clinical practice guidelines: liver transplantation. Journal of Hepatology 2016;64:433-85.

[66] Ramirez CB, Doria C, Frank AM, et al. Completely steroid-free immunosuppression in liver transplantation: a randomized study. Clinical Transplantation 2013;27:463-71.

[67] Weiler N, Thrun I, Hoppe-Lotichius M, et al. Early steroid-free immunosuppression with FK506 after liver transplantation: long-term results of a prospectively randomized double-blinded trial. Transplantation 2010;90:1562-6.

[68] Kato T, Gaynor JJ, Yoshida H, et al. Randomized trial of steroid-free induction versus corticosteroid maintenance among orthotopic liver transplant recipients with hepatitis $C$ virus: impact on hepatic fibrosis progression at one year. Transplantation 2007;84:829-35.

[69] Llado L, Xiol X, Figueras J, et al. Immunosuppression without steroids in liver transplantation is safe and reduces infection and metabolic complications: results from a prospective multicenter randomized study. Journal of Hepatology 2006;44:710-6.

[70] Manousou P, Samonakis D, Cholongitas E, et al. Outcome of recurrent hepatitis $C$ virus after liver transplantation in a randomized trial of tacrolimus monotherapy versus triple therapy. Liver Transplantation 2009;15:1783-91.

[71] Duvoux C, Pageaux GP. Immunosuppression in liver transplant recipients with renal impairment. Journal of Hepatology 2011;54:1041-54.

[72] De Simone P, Nevens F, De Carlis L, et al. Everolimus with reduced tacrolimus improves renal function in de novo liver transplant recipients: a randomized controlled trial. American Journal of Transplantation 2012;12:3008-20.

[73] Kong Y, Wang D, Shang Y, et al. Calcineurin-inhibitor minimization in liver transplant patients with calcineurin-inhibitor-related renal dysfunction: a meta-analysis. PLoS One 2011;6:e24387.

[74] Sterneck M, Kaiser GM, Heyne N, et al. Everolimus and early calcineurin inhibitor withdrawal: 3-year results from a randomized trial in liver transplantation. American Journal of Transplantation 2014;14:701-10.

[75] Trunecka P, Klempnauer J, Bechstein WO, et al. Renal function in de novo liver transplant recipients receiving different prolonged-release tacrolimus regimens-the DIAMOND study. American Journal of Transplantation 2015; 15:1843-54.

[76] De Simone P, Fagiuoli S, Cescon M, et al. Use of everolimus in liver transplantation: recommendations from a working group. Transplantation 2016 [Epub ahead of print].

[77] Dumortier J, Gagnieu MC, Salandre J, et al. Conversion from mycophenolate mofetil to enteric-coated mycophenolate sodium in liver transplant patients presenting gastrointestinal disorders: a pilot study. Liver Transplantation 2006; 12:1342-6.

[78] Lopez-Solis R, Devera M, Steel J, et al. Gastrointestinal side effects in liver transplant recipients taking enteric-coated mycophenolate sodium vs. mycophenolate mofetil. Clinical Transplantation 2014;28:783-8.

[79] Tang CY, Shen A. Wei XF, et al. Everolimus in de novo liver transplant recipients: a systematic review. Hepatobiliary and Pancreatic Diseases International 2015;14:461-9.

[80] Wang Z, He JJ, Liu XY, et al. The evaluation of enteric-coated mycophenolate sodium in cardiac deceased donor liver transplant patients in China. Immunopharmacology and Immunotoxicology 2015;37:508-12.

[81] Liu Z, Chen Y, Tao R, et al. Tacrolimus-based versus cyclosporinebased immunosuppression in hepatitis C virus-infected patients after liver transplantation: a meta-analysis and systematic review. PLoS One 2014;9:e107057

[82] Klintmalm GB, Davis GL, Teperman L, et al, A randomized, multicenter study comparing steroid-free immunosuppression and standard immunosuppression for liver transplant recipients with chronic hepatitis C. Liver Transplantaion 2011;17:1394-403.

[83] Duvoux C, Toso C. mTOR inhibitor therapy: does it prevent HCC recurrence after liver transplantation? Transplantation Reviews (Orlando) 2015;29:168-74.

[84] Rodriguez-Peralvarez M, De La Mata M, Burroughs AK. Liver transplantation: immunosuppression and oncology. Current Opinion in Organ Transplantation 2014;19:253-60.

[85] Geissler EK, Schnitzbauer AA, Zulke C, et al. Sirolimus use in liver transplant recipients with hepatocellular carcinoma: a randomized, multicenter, openlabel phase 3 trial. Transplantation 2016;100:116-25.

[86] Fischer L, Klempnauer J, Beckebaum S, et al. A randomized, controlled study to assess the conversion from calcineurin-inhibitors to everolimus after liver transplantation-PROTECT. American Journal of Transplantation 2012;12:1855-65.

[87] Finkenstedt A, Graziadei IW, Oberaigner W, et al. Extensive surveillance promotes early diagnosis and improved survival of de novo malignancies in liver transplant recipients. American Journal of Transplantation 2009;9:2355-61.

[88] Anastacio LR, Ferreira LG, Ribeiro Hde S, et al. Metabolic syndrome after liver transplantation: prevalence and predictive factors. Nutrition 2011;27:931-7. 
[89] Fussner LA, Heimbach JK, Fan C, et al. Cardiovascular disease after liver transplantation: when, what, and who is at risk. Liver Transplantation 2015;21:889-96.

[90] Watt KD. Metabolic syndrome: is immunosuppression to blame? Liver Transplantation 2011;17(Suppl. 3):S38-42.

[91] Baid S, Cosimi AB, Farrell ML, et al. Posttransplant diabetes mellitus in liver transplant recipients: risk factors, temporal relationship with hepatitis $C$ virus allograft hepatitis, and impact on mortality. Transplantation 2001;72:1066-72.

[92] Heisel O, Heisel R, Balshaw R, et al. New onset diabetes mellitus in patients receiving calcineurin inhibitors: a systematic review and meta-analysis. American Journal of Transplantation 2004;4:583-95.

[93] Richards J, Gunson B, Johnson J, Neuberger J. Weight gain and obesity after liver transplantation. Transplant International 2005;18:461-6.

[94] Kozlowski T, Rubinas T, Nickeleit V, et al. Liver allograft antibody-mediated rejection with demonstration of sinusoidal C4d staining and circulating donor-specific antibodies. Liver Transplantation 2011;17:357-68.

[95] Teperman L, Moonka D, Sebastian A, et al. Calcineurin inhibitor-free mycophenolate mofetil/sirolimus maintenance in liver transplantation: the randomized spare-the-nephron trial. Liver Transplantation 2013;19:675-89.

[96] Dannhorn E, Cheung M, Rodrigues S, et al. De novo use of generic tacrolimus in liver transplantation-a single center experience with one-yr follow-up. Clinical Transplantation 2014;28:1349-57.

[97] Taube D, Jones G, O’Beirne J, et al. Generic tacrolimus in solid organ transplantation. Clinical Transplantation 2014;28:623-32.

[98] Vollmar J, Bellmann MC, Darstein F, et al. Efficacy and safety of a conversion from the original tacrolimus and mycophenolate mofetil to the generics tacpan and mowel after liver transplantation. Journal of Drug Design, Development and Therapy 2015;9:6139-49.

[99] Taner T, Gandhi MJ, Sanderson SO, et al. Prevalence, course and impact of HLA donor-specific antibodies in liver transplantation in the first year. American Journal of Transplantation 2012;12:1504-10.

[100] Del Bello A, Congy-Jolivet N, Danjoux M, et al. De novo donor-specific antiHLA antibodies mediated rejection in liver-transplant patients. Transplant International 2015;28:1371-82.

[101] O'Leary JG, Kaneku H, Demetris AJ, et al. Antibody-mediated rejection as a contributor to previously unexplained early liver allograft loss. Liver Transplantation 2014;20:218-27.

[102] O'Leary JG, Kaneku H, Banuelos N, et al. Impact of IgG3 subclass and C1qfixing donor-specific HLA alloantibodies on rejection and survival in liver transplantation. American Journal of Transplantation 2015;15:1003-13.
[103] Ruiz R, Tomiyama K, Campsen J, et al. Implications of a positive crossmatch in liver transplantation: a 20-year review. Liver Transplantation 2012;18:455-60.

[104] Troxell ML, Lanciault C. Practical applications in immunohistochemistry: evaluation of rejection and infection in organ transplantation. Archives of Pathology and Laboratory Medicine 2016;140:910-25.

[105] Boleslawski E, Benothman S, Grabar S, et al. CD25, CD28 and CD38 expression in peripheral blood lymphocytes as a tool to predict acute rejection after liver transplantation. Clinical Transplantation 2008;22:494-501.

[106] Rodrigo E, Lopez-Hoyos M, Corral M, et al. ImmuKnow as a diagnostic tool for predicting infection and acute rejection in adult liver transplant recipients: a systematic review and meta-analysis. Liver Transplantation 2012;18:1245-53.

[107] Rodriguez-Peralvarez M, Germani G, Tsochatzis E, et al. Predicting severity and clinical course of acute rejection after liver transplantation using blood eosinophil count. Transplant International 2012;25:555-63.

[108] Pons JA, Ramirez P, Revilla-Nuin B, et al. Immunosuppression withdrawal improves long-term metabolic parameters, cardiovascular risk factors and renal function in liver transplant patients. Clinical Transplantation 2009;23:329-36.

[109] Tisone G, Orlando G, Cardillo A, et al. Complete weaning off immunosuppression in HCV liver transplant recipients is feasible and favourably impacts on the progression of disease recurrence. Journal of Hepatology 2006;44:702-9.

[110] Benitez C, Londono MC, Miquel R, et al. Prospective multicenter clinical trial of immunosuppressive drug withdrawal in stable adult liver transplant recipients. Hepatology 2013;58:1824-35.

[111] Bohne F, Martinez-Llordella M, Lozano JJ, et al. Intra-graft expression of genes involved in iron homeostasis predicts the development of operational tolerance in human liver transplantation. Journal of Clinical Investigation 2012;122:368-82.

[112] Li L, Wozniak LJ, Rodder S, et al. A common peripheral blood gene set for diagnosis of operational tolerance in pediatric and adult liver transplantation. American Journal of Transplantation 2012;12:1218-28.

[113] Oellerich M, Walson PD, Beck J, et al. Graft-derived cell-free dna as a marker of transplant graft injury. Therapeutic Drug Monitoring 2016;38(Suppl. 1):S75-9. 